\title{
Levi-Civita Cylinders with Fractional Angular Deficit
}

\author{
J.P. Krisch and E.N. Glass \\ Department of Physics, University of Michigan, Ann Arbor, MI 48109
}

(Dated: 02 March 2011)

\begin{abstract}
The angular deficit factor in the Levi-Civita vacuum metric has been parametrized using a Riemann-Liouville fractional integral. This introduces a new parameter into the general relativistic cylinder description, the fractional index $\alpha$. When the fractional index is continued into the negative $\alpha$ region, new behavior is found in the Gott-Hiscock cylinder and in an Israel shell.
\end{abstract}

\section{INTRODUCTION}

The 1917 Levi-Civita ${ }^{1]}$ solution is a standard vacuum exterior for cylindrical matter distributions. The metric can be written in terms of two parameters: mass density $\sigma$ and angular deficit $b$

$$
d s^{2}=-r^{4 \sigma} d t^{2}+r^{-4 \sigma}\left[r^{8 \sigma^{2}}\left(d r^{2}+d z^{2}\right)+b^{2} r^{2} d \phi^{2}\right]
$$

The interior matches add a mass/length parameter, $\lambda$, to the description of the complete solution. Currently 3-dim cylindrical matter distributions with positive density are believed to provide a good description for $0 \leq \sigma<1 / 2 .{ }^{2]-[17]}$ The literature has tended to focus on the $(\sigma, \lambda)$ ranges but one additional feature in many of the interior solutions is the boundary location and size that varies as a function of the density. The size of the circumference remains finite even as the density increases and a coordinate radius blows up. ${ }^{6}$, 7] This suggests that the boundary circumference is an interesting parameter to include in the analysis of cylinder solutions.

In this paper we consider a circumference calculated using a Riemann-Liouville fractional integral. This introduces a new parameter into the cylinder description, the fractional index $\alpha$. The circumference calculated from the Levi-Civita metric involves the angular deficit factor. A fractional circumference can relate the physical behavior of the angular deficit to a mathematical framework through $b(\alpha)$. Matches to an interior will provide a fractional parametrization of all of the cylinder parameters. An unexpected result of the fractional extension is a new family of solutions occuring at one of the angular deficit embedding $[6]$ 
transition points.

In the next section we briefly review the literature leading to limits on the range of $\sigma$, and develop the fractional circumference. The idea is applied to the $\sigma=0$ Gott-Hiscock ${ }^{6}$, 7] constant density string solution, providing a parametrization of $\lambda$. The method is extended to a $\sigma \neq 0$ shell $[16]$ in the third part of the paper and the behavior of $\lambda$ and $\sigma$ are linked. An Appendix introducing some of the basic fractional integral definitions is included.

\section{CYLINDER SOLUTIONS}

\section{The range of $\sigma$}

The Riemann tensor of metric (11) is zero for $\sigma=(0,1 / 2)$ and is singular as $r$ approaches zero

$$
\lim _{r \rightarrow 0}\left[R_{a b c d} R^{a b c d}=\frac{64 \sigma^{2}\left(4 \sigma^{2}-2 \sigma+1\right)(2 \sigma-1)^{2}}{r^{4\left(4 \sigma^{2}-2 \sigma+1\right)}}\right] \rightarrow \infty .
$$

The literature contains a number of discussions that provide interior matter distributions motivating some of this behavior. An infinite matter cylinder is often considered because, in the small $\sigma$ limit, a test particle at rest experiences an acceleration $\ddot{r}=-2 \sigma / r$. This is the Newtonian acceleration for a particle a distance $r$ from a line source of mass/length $(\sigma)$. 13] In addition, through metric matching, $\sigma$ can often be related to the matter stress-energy coming from the field equations. The identification is not absolute. The constant density Gott-Hiscock solutions ${ }^{6}$, 7] and the $\mathrm{U}(1)$ string solutions of Garfinkle ${ }^{18]}$ both use the $\sigma=0$ Levi-Civita vacuum. The exact cylindrical solid matter solutions that can be matched to the Levi-Civita vacuum indicate a restricted range for $\sigma, 0 \leq \sigma<1 / 2$. Some of the 3-dim matter solutions that can be matched to $\sigma=0$ also show restricted ranges in the cylinder mass/length, $\lambda$, following from the field equations. Israel shells bounding vacuum Levi-Civita and a second vacuum interior indicate a broader range. $\frac{16}{[6]}$

One of the early investigations of static cylindrical solutions, due to Marder ${ }^{2]}$, directly examined the relation between $\sigma$ and the cylinder $\lambda$ by matching to a Levi-Civita vacuum with no angular deficit. Krori and Paul ${ }^{3}$ used Marder's solution to establish a limit $\sigma<1 / 2$. For general $\sigma_{\text {p pavidson }}$ [ developed a set of static cylindrical solutions which were used by Bonnor et al 9,10$]$ to study the possible range of $\sigma$. One of the motivating questions for this discussion was the zero Riemann tensors for $\sigma=(0,1 / 2)$. The $\sigma=0$ flat spacetime 
was interpreted as a matter-free vacuum but the $\sigma=1 / 2$ case was left an open question. Philbin ${ }^{13]}$ extended cylindrical solutions into the negative $\sigma$ region and suggested that the endpoints $|\sigma|=1 / 2$ described planar rather than cylindrical matter distributions. Based on the behavior of gyros orbiting a cylindrical mass distribution, Herrera, Ruifernández, and Santos ${ }^{14]}$ also suggested that $\sigma=1 / 2$ described a planar mass. In a later paper ${ }^{15]}$, Herrera et al treated the $\sigma=1 / 2$ transition point in greater detail and, with the $(\varphi, z)$ coordinates taken as interchangeable to include possible planar topologies, suggested models for the coordinate range $0 \leq \sigma<\infty$.

Some of the work on static cylinders is embedded in discussions of rotating cylinders with the parameter and density limits appearing for both cases. For example, one of the early indications of a restricted relativistic density range was the rotating dust cylinder of Vishveshwara and Winicour. ${ }^{4]}$ This interesting paper contains an expression for $\lambda$ related to an angular deficit and identifies the $b=0$ deficit factor as a critical limit related to a rotating column with $\lambda \leq 1 / 4$. Building on this work, Lathrop and Orsene ${ }^{[5]}$ considered a cylinder with two counter-rotating dust currents and, for this matter source, dupicated the Vishveshwara-Winicour ${ }^{[4]}$ density limit, as did later work by da Silva et al. 12]

\section{Fractional Circumference}

A fractional integral is a function convolution over a range. The fractional integral used to calculate the fractional circumference is the Riemann-Liouville form [19, 20]

$$
I^{-\alpha}[f(x)]=\frac{1}{\Gamma(\alpha)} \int_{0}^{x} f(y)(x-y)^{\alpha-1} d y
$$

where $\alpha$ is the fractional order, $\operatorname{Re}(\alpha)>0$. A fractional circumference is calculated by integrating $\sqrt{g_{\phi \phi}}$ around the circle. $x$ is identified as $\phi$ with the circumference following in the $\phi \rightarrow 2 \pi$ limit. For the base metric, consider a $3+1$ Minkowski metric with cylindrical coordinates $(t, r, \phi, z)$.

$$
d s^{2}=-d t^{2}+d r^{2}+r^{2} d \phi^{2}+d z^{2}
$$

The usual circumference of a circle in the $(r, \phi)$ plane for $r=r_{0}$ is $C=2 \pi r_{0} . r_{0}$ is the coordinate radius. A fractional circumference for the same coordinate radius is

$$
C^{(\alpha)}=\frac{1}{\Gamma(\alpha)} \lim _{\phi \rightarrow 2 \pi} \int_{0}^{\phi} \sqrt{g_{\phi \phi}}(\phi-y)^{\alpha-1} d y=2 \pi r_{0} \frac{(2 \pi)^{\alpha-1}}{\Gamma(1+\alpha)} .
$$


$\alpha=1$ gives the usual circumference. For $0<\alpha<1$, the circumference is less than the

usual $2 \pi r_{0}$ and for $\alpha>1$ it is larger. The methods of fractional calculus ${ }^{21}$ 23] have been very successful in modeling transport processes with anomalous microscopic time and/or

spatial structure. 24, 25] One could expect that the density of matter distributions interior to the boundary would reflect the transport processes responsible for their growth. In this case, it is the variation in the circumference with $\alpha$ that we wish to link to matter distributions with an angular deficit, with the fractional variation in circumference reflecting a fractional surface matter distribution. If coordinate ranges are not imposed, the angular deficit can be transformed away. Here it is strongly linked to a physical description with the usual imposed angular coordinate ranges. Bonner $\underline{26]}$ noted that the angular deficit parameter, $b$, determines the topology of the manifold covered by metric (11) and cannot be removed by scale transformations. Angular deficit is a topological defect like the gravitational AharonovBohm effect discussed by Jensen and Kučera 27$]$

In the following, we apply the fractional circumference to the parameters of the $\sigma=0$ Gott-Hiscock constant density string.

\section{The Gott-Hiscock String}

The Gott-Hiscock solution describes an interior constant density, $\delta$, cylinder matched to a $\sigma=0$ Levi-Civita vacuum with angular deficit $b$. The matter $(-)$ and vacuum $(+)$ metrics are

$$
\begin{aligned}
& d s_{-}^{2}=-c^{2} d t^{2}+d \rho^{2}+\left[\frac{\sin (\delta \rho)}{\delta}\right]^{2} d \phi^{2}+d z^{2} \\
& d s_{+}^{2}=-c^{2} d t^{2}+d r^{2}+b^{2} r^{2} d \phi^{2}+d z^{2}
\end{aligned}
$$

The string radii in the interior and exterior are $\rho_{0}$ and $r_{0}$. The matching relations are $\sin \left(\delta \rho_{0}\right)=\delta b r_{0}$, and $b=\cos \left(\delta \rho_{0}\right)$ and the mass per unit length, $\lambda$, of the constant density string is $\lambda=(1-b) / 4$. The angular deficit/excess associated with $b$ is $\Delta \phi=2 \pi(1-b)=8 \pi \lambda$. The allowed range of $b$ is $-1 \leq b \leq 1$ and the positive $\lambda$ range is $0 \leq \lambda \leq 1 / 2$.

The boundary match provides an expression for the coordinate radius, $r_{0}$ and associated 
FIG. 1: embedding diagrams for the GH string

circumference

$$
\begin{aligned}
& r_{0}=\frac{\tan \left(\delta \rho_{0}\right)}{\delta} \\
& C=2 \pi r_{0} b=2 \pi \frac{\sin \left(\delta \rho_{0}\right)}{\delta}
\end{aligned}
$$

A $(r, \phi)$ cross section of the exterior vacuum is regarded as a circle with a missing pie slice. $r_{0} b$ is the radius of the equivalent closed circle. The matching relations indicate that $r_{0}$ approaches $\infty$ as $\delta \rho_{0} \rightarrow \pi / 2$, while $b=0$ and the circumference takes its maximum value. Gott [ $[$ has motivated this behavior by embedding the $(t, z)=$ const Levi-Civita vacuum into a 3-dim metric with coordinates $\left(w, r^{\prime}=b r, \phi\right)$, the embedding relation between $w$ and $r^{\prime}$ defining a cone in the 3-dim space. This embedding picture is illustrated in the sketches of Figure 1. The shaded region represents a matter cap in the interior. The sketches illustrate the entire $b$ range. In the integer model, as the density increases from $\lambda=0(b=1)$ to $\lambda=1 / 4$ the upper sides of the cone tilt outward to form a $b=0$ cylinder. As the mass continues to increase, the center moves out into the vacuum, the lower sides tilting in to form an upward pointing cone. Conventionally this is associated with negative $b$, angular excess and a quasi-regular singularity $\underline{4}]$ in the vacuum.

The circle defined by the matter/vacuum match has an effective radius $r_{0} b$ and is clearly maximum for the $b=0$ cylinder.

\section{Fractional Angular Deficit}

The metric circumference of the string in the exterior spacetime is $2 \pi b r_{0}$ where $b r_{0}$ is the effective radius of the closed circle. Equating this to the $\alpha>0$ fractional circumference one 
FIG. 2: b vs $\alpha, \alpha>-0.5$

finds a fractional angular deficit factor

$$
\begin{aligned}
2 \pi b r_{0} & =2 \pi r_{0} \frac{(2 \pi)^{\alpha-1}}{\Gamma(1+\alpha)} \\
b(\alpha) & =\frac{(2 \pi)^{\alpha-1}}{\Gamma(1+\alpha)}
\end{aligned}
$$

$\alpha=1$ can be identified with $b=1$, the vacuum case. Fig.2 shows $b(\alpha)$ over the range $-0.5 \leq \alpha \leq 1$. $b$ decreases toward 0 as $\alpha \rightarrow 0$ and $\lambda$ moves toward the cone $\rightarrow$ cylinder transition value, $\lambda=1 / 4$.

In this region, $b r_{0}$, the effective radius in the circumference, increases from its vacuum 0 to its maximum value $b r_{0}=1 / \delta$. This behavior is implicit in the embedding diagrams. The value of $\alpha$ follows from $\delta \rho_{0}=\sin ^{-1}\left(b r_{0}\right)$. In the $\alpha$ region shown in Fig.2, the fractional index is only another parameter that provides a mathematical base for $b$. However, while the fractional circumference is strictly defined for $\alpha>0$, the graph shows a continuation across the origin into the undefined negative alpha region. The interpretation of negative $\alpha$ will come from the string models it parametrizes. In Fig.2, there is no identification of an $\alpha$ parametrization for $b=0$ until the alpha range is extended further along the negative axis. This extension is shown in Fig. 3. There are a sequence of points of decreasing amplitude, oscillating around $b=0$. The fractional string model has only a small extension into the negative $b$ region with its vacuum quasi-regular singularity.

The identification of a fractional form for $b$ can be extended to the string mass/length showing $\lambda$ oscillations about the cone $\rightarrow$ cylinder critical value $\lambda=1 / 4$. 
FIG. 3: b vs $\alpha,-0.5<\alpha$

FIG. $4: \lambda$ vs $\alpha,-0.5<\alpha$

The mass/length is shown in Figures 4 and 5. Fig.4 supports the identification of $b=1$, $\alpha=1$ with a zero string vacuum and Fig.5 shows the expected oscillations in the string mass/length about $\lambda=1 / 4$. The string coordinate radius, $r_{0}=\tan \left(\delta \rho_{0}\right) / \delta$, blows up at $\delta \rho_{0}=\pi / 2$ but the product $b r_{0}$ entering into the circumference is finite, showing small oscillations around $r_{0} b=1 / \delta$, the effective radius for the cylindrical embedding. This example used a 3-dim matter distribution inside the cylinder. The next example considers a cylindrical shell. 
FIG. 5: $\lambda$ vs $\alpha, \alpha<-0.75$

\section{CYLINDRICAL SHELLS}

$2+1$ cylindrical shells have also been used to explore the static parameter/density limits.

A sequence of cylindrical shells was first suggested by Marder [2] and Wang et al [16] discussed a shell with stress-energy resulting from a jump from exterior Levi-Civita to an interior Minkowski. A simple cylindrical model with non zero $\sigma$ has exterior $(+)$ and interior (-) metrics

$$
\begin{aligned}
& d s_{+}^{2}=-\left(r / r_{0}\right)^{4 \sigma} d t^{2}+\left(r / r_{0}\right)^{-4 \sigma}\left[\left(r / r_{0}\right)^{8 \sigma^{2}}\left(d r^{2}+d z^{2}\right)+b^{2} r^{2} d \phi^{2}\right] \\
& d s_{-}^{2}=-d t^{2}+d \rho^{2}+d z^{2}+\rho^{2} d \phi^{2}
\end{aligned}
$$

The metric match across a surface $\left(r_{0}, \rho_{0}\right)$ is

$$
\rho_{0}=b r_{0}
$$

This matching relation suggests that the circumference is bounded as $b$ and $r_{0}$ change. The extrinsic curvatures are $K_{a b}^{ \pm}=n_{a ; b}^{ \pm}=-\Gamma_{a b}^{ \pm} r n_{r}^{ \pm}=\partial_{r} g_{a b} / 2$ at $r=r_{0}$. The shell has stressenergy, $S_{a b}$, related to the extrinsic curvature jump $<K_{a b}>=K_{a b}^{+}-K_{a b}^{-},<K>=<K_{a}^{a}>$, 
across the boundary. ${ }^{28,}$, 29] Calculating the stress-energy one finds, with $\digamma(\sigma)=1-4 \sigma+4 \sigma^{2}$

$$
\begin{aligned}
-8 \pi S_{j}^{i} & =<K_{j}^{i}>-h_{j}^{i}<K> \\
-8 \pi S_{t}^{t} & =\frac{1-b \digamma(\sigma)}{b r_{0}} \\
-8 \pi S_{\phi}^{\phi} & =-4 \sigma^{2} / r_{0} \\
-8 \pi S_{z}^{z} & =\frac{1-b}{b r_{0}}
\end{aligned}
$$

Using the same simple density integral to calculate $\lambda$ as was used in the Gott-Hiscock string, one finds the stress-energy parameters

$$
\begin{aligned}
4 \lambda & =1-b \digamma(\sigma) \\
8 \pi P_{\phi} & =4 \sigma^{2} / r_{0} \\
8 \pi P_{z} & =\frac{b-1}{b r_{0}} \\
8 \pi \varepsilon & =\frac{1-b \digamma(\sigma)}{b r_{0}}
\end{aligned}
$$

The general equation of state 11,17$]$ is

$$
\varepsilon+P_{\phi}+P_{z}=4 \sigma / r_{0}
$$

Looking first at $\sigma=0$, the usual stiff string equation of state is found. This is an interesting comparison case to the GH string. It does describe a constant density object with an axial tension and $\lambda$ but the limits are set by the angular deficit factor, not by trignometric limits. Using ad hoc values $b=(1,0,-1)$, we have $4 \lambda=(0,1,2)$. Thus we have the same set of boundary values as the GH string. With $b=(2 \pi)^{\alpha-1} / \Gamma(1+\alpha)$ the mass/length has the same form as for the GH string

$$
4 \lambda=1-\frac{(2 \pi)^{\alpha-1}}{\Gamma(1+\alpha)}
$$

and the same density oscillations around $b=0$ are present. A large difference between the two cases is that $b$ is not limited to a unit range, without the GH identification of $b$. The current limits on $\sigma$ would restrict $b$. For $\sigma \neq 0$ the immediate observations are, that for fixed radius, $\sigma$ determines the tangential stress while the axial stress is determined by $b$. Links between $\sigma$ and $b$ follow from assumptions on the partial equations of state linking $\varepsilon$ and individual stresses, or on relations between the stresses. For example, a shell with uniform stress, $P_{\phi}=P_{z}$ imposes $b=1 /\left(1-4 \sigma^{2}\right)$. In this model, isotropic stress is associated 
with negative mass/length. A physical solution will have anisotropic stresses, avoiding a singularity at $\sigma=1 / 2$.

\section{CONCLUSION}

In this paper we have examined a fractional parametrization $b(\alpha)$ of the angular deficit factor. The original motivation was to match the physical behavior of the angular deficit to a mathematical framework providing a fractional parametrization of cylinder parameters. For the Gott-Hiscock cylinder, we found new behavior when the angular deficit factor is continued from a vacuum with a conical defect across the embedding cylinder, $b=0$, into a vacuum with a quasi-regular singularity. The behavior may have implications for the stability of the cylinder solutions in the $b$ parameter range. 30, 31] This is under investigation. The models considered in the paper are for the Levi-Civita $\sigma$ in the range $0 \leq \sigma<1 / 2$. The applicability of a fractional angular deficit as developed here, depends on an angular coordinate with an associated circumference. Herrera et al $\left.{ }^{15}\right]$ used coordinates $(\varphi, z)$ with ranges $-\infty$ to $\infty$, imposing a periodicity, $\varphi=\varphi+2 \pi$ on the angular coordinate for $\sigma<1 / 2$. Our result applies in that region. Their results seem to imply that $\sigma=1 / 2$ is associated with a planar topology and that there is no appropriate angular coordinate. For $\sigma>1 / 2$ they suggest that the Levi-Civita coordinates $(\varphi, z)$ are interchanged, with $z$ becoming the periodic measure, $z=z+z_{0}$. The circumference for this $\sigma$ region would be

$$
C^{(\alpha)}=\frac{1}{\Gamma(\alpha)} \lim _{z \rightarrow z_{0}} \int_{0}^{z} \sqrt{g_{z z}}(z-y)^{\alpha-1} d y=\frac{\left(z_{0}\right)^{\alpha}}{\Gamma(1+\alpha)}
$$

It is also possible that both $z$ and $\varphi$ are periodic in this region suggesting a toroidal matter distribution rather than a cylindrical one. This extended view would allow two fractional circumferences to be included in the modeling. The complete topological description of Levi-Civita matched matter distributions, and its fractional extensions, still have many open questions. The infinite cylinder, both static and rotating, continues to be an interesting and useful structure in general relativity: The parameter effects due to including a cosmological constant in Levi-Civita ${ }^{\text {32, 33] }}$ are-beginning to be examined ${ }^{34]}$ and, with the recent connections to braneworld cosmologies 35, 36], relativistic cylinders have acquired new physical relevance. 


\section{Appendix A: FRACTIONAL CALCULUS}

Fractional calculus was invented in 1695 when L'Hopital ${ }^{19},[20]$ asked Leibniz about the meaning of his notation $\frac{d^{n} y}{d x^{n}}$ for $n=1 / 2$. This question, also asked by Bernoulli defined the idea of a fractional derivative and L'Hopital's question about derivatives has developed into a fractional calculus, a framework for integrals and derivatives of non-integer order. Euler made the first real contribution to the development of fractional calculus with his 1738 proof that the fractional derivative of $x^{n}$ was meaningful. $\underline{37}$ The first contribution using the idea is credited to Abel for his 1823 work on the tautochrone. 20] The idea of fractional calculus has attracted the attention of many of the same people that are associated with the development of physics and general relativity: Lagrange, Laplace, Fourier, Liouville, Riemann and Weyl $\left.{ }^{19}, 20\right]$ among others. There are several definitions of fractional integrals depending on the integral limits. The Riemann form integrates from $c$ to $x$, The Liouville version from $-\infty$ to $x$, and in this paper the Riemann-Liouville form is used from 0 to $x$. 20]

$$
I^{-\alpha}[f(x)]=\frac{1}{\Gamma(\alpha)} \int_{0}^{x} f(y)(x-y)^{\alpha-1} d y
$$

$\alpha$ is the fractional order, $\operatorname{Re}(\alpha)>0$. There is also a Weyl form for the fractional integral with a $+\infty$ limit.

In order for the Riemann-Liouville integral to converge it is necessary that $f(x)$ satisfy the condition

$$
f\left(x^{-1}\right)=O\left(x^{1-\delta}\right) \quad \delta>0
$$

Functions obeying this condition are Riemann class; for example, the function $x^{b}, b>-1$ is a Riemann class function. The Weyl integral is useful for functions with the convergence property

$$
f(-x)=O\left(x^{-\nu-\delta}\right) \delta>0, x \rightarrow \infty
$$

Functions satisfying this condition are said to be of Liouville class; for example, $x^{-b}$ with $b>\nu>0$, is a Liouville function. The allowable parameter range eliminates constants from this class. There can be overlap between classes.

The evaluation of fractional integrals uses the beta and gamma functions. The gamma 
function is defined by

$$
\begin{aligned}
\Gamma(x) & :=\int_{0}^{\infty} e^{-t} t^{\alpha-1} d t, \quad x>0 \\
\Gamma(x+1) & =x \Gamma(x), \quad x>0
\end{aligned}
$$

For positive integer $n, \Gamma(n+1)=n$ !. $\Gamma(x)$ is extended to negative $x$ by the functional equations

$$
\begin{aligned}
\Gamma(x) & :=\frac{\Gamma(x+1)}{x}, \quad-1<x<0 \\
\Gamma(x) & :=\frac{\Gamma(x+2)}{x(x+1)}, \quad-2<x<-1 \\
\Gamma(x) & :=\frac{\Gamma(x+n)}{x(x+1) \cdots(x+n-1)}, \quad-n<x<-n+1
\end{aligned}
$$

The beta function is defined as

$$
B(z, w)=\int_{0}^{1} x^{z-1}(1-x)^{w-1} d x
$$

It is related to the gamma function

$$
B(z, w)=\frac{\Gamma(z) \Gamma(w)}{\Gamma(z+w)}
$$

For example, the fractional integral of $f(x)=1$ is

$$
D_{0}^{-\nu}[1]=\frac{1}{\Gamma(\nu)} \int_{0}^{x}(x-y)^{(\nu-1)} d y
$$

Comparing with the definitions of the beta and gamma functions, we see the fractional integral of 1 is given by

$$
D_{0}^{-\nu}[1]=\frac{\Gamma(1)}{\Gamma(\nu+1)} x^{\nu}
$$

Another example is the fractional integral of $f(x)=x^{\alpha}$. Using the definitions the integral becomes

$$
D_{0}^{-\nu}\left[x^{\alpha}\right]=\frac{\Gamma(1+\alpha)}{\Gamma(\alpha+\nu+1)} x^{\nu+\alpha}
$$

For $\nu=1$, this becomes $x^{\alpha+1} /(\alpha+1)$, the usual integral of $x^{\alpha}$. For $\alpha=-1$, the gamma function in the numerator is singular so the fractional integral of $1 / x$ is not defined. The 
analytic continuation used in the paper assigned meaning to the singular points of the gamma function.

[1] T. Levi-Civita, Rend. Acc. Liniei 27, 183 (1917).

[2] L. Marder, Proc. Roy. Soc. Lond. A 244, 524 (1958). Gravitational waves in General Relativity

[3] K.D. Krori and B.B. Paul, J. Phys. A 10, 25 (1977). Interior solution of a tangentialy stressed cylinder in general relativity.

[4] C.V. Vishveshwara and J. Winicour, J. Math. Phys. 18, 1280 (1977). Relativisticaly rotating dust cylinders.

[5] J.D. Lathrop and M.S. Orsene, J. Math. Phys. 21, 152 (1980). Dust Cylinders in Static Spacetimes.

[6] J. Richard Gott III, Astrophys. J. 288, 422 (1985). Gravitational Lensing Effects of Vacuum Strings: Exact Solutions

[7] W.A. Hiscock, Phys. Rev. D 31, 3288 (1985). Exact Gravitational Field of a String

[8] W. Davidson, Gen. Rel. Gravit. 22, 553 (1990). A Solution for a Family of Perfect Fluid Cylinders in General Relativity.

[9] W.B. Bonnor and M.A. Martins, Class. Quan. Grav. 8, 727 (1991). The interpretation of some static vacuum metrics.

[10] W.B. Bonnor and W. Davidson, Class. Quan. Grav. 9, 2065 (1992). Interpreting the LeviCivita vacuum metric.

[11] G. Clement, Phys. Rev. D 50, 777 (1994), Hollow cosmic string: the general-relativistic hollow cylinder.

[12] M.F.A. da Silva, L. Herrera, F. M. Paiva and N.O. Santos, J. Math. Phys. 36, 3625 (1995). The Levi Civita Spacetime

[13] T.G. Philbin, Class. Quan. Grav. 13, 1217 (1996). Perfect-fluid cylinders and walls-sources for the Levi-Civita Spacetime.

[14] L. Herrera, J. Ruifernández and N.O. Santos, Gen. Rel. Gravit. 33, 515 (2001). The Static Cylinder, Gyroscopes, the C-metric and All That.

[15] L. Herrera, N.O. Santos, A.F.F. Teixeira, and A.Z. Wang, Class. Quan. Grav. 18, 3847 (2001). On the interpretation of cylindrically symmetric Levi-Civita spacetime for $0 \leq \sigma<\infty$ 
[16] A.Z. Wang, M.F.A. da Silva and N.O Santos, Class. Quan. Grav. 14, 2417 (1997). On parameters of the Levi-Civita solution

[17] J. Bicak, T. Ledvinka, B.G. Schmidt and M. Zofka, Class. Quan. Grav. 21, 1538 (2004). Static fluid cylinders and their fields: global solutions.

[18] D. Garfinkle, Phys. Rev. D 32, 1323 (1985). General relativistic strings

[19] K.B. Oldham and J. Spanier, The Fractional Calculus, (Academic Press, New York, 1974).

[20] K.S. Miller and B. Ross, An Introduction to the Fractional Calculus and Fractional Differential Equations (John Wiley \& Sons, New York, 1993), p. 23

[21] F. Mainardi, Fractals and Fractional Calculus in Continuum Mechanics, edited by A. Carpinteri and F. Mainardi (Springer, New York, 1997).

[22] I. Podlubny, Fractional Differential Equations (Academic Press, New York, 1999).

[23] P.L. Butzer and U. Westphal, Applications of Fractional Calculus in Physics, Ed.R. Hilfer (World Scientific, Singapore, 2000).

[24] I.M. Sokolov, J. Klafter and A. Blumen, Physics Today, 55, 48 (2002). Fractional Kinetics

[25] E. Scalas, R. Gorenflo, F. Mainardi, and M. Raberto, International Workshop on Scaling and Disordered Systems, Paris, France, 13-14 April 2000, Scaling and Disordered Systems, (F. Family et al. eds.), pp. 281-289,( World Scientific, 2002), arXiv:cond-mat/0210166 Revisiting the Derivation of the Fractional Diffusion Equation

[26] W.B. Bonner, J. Phys. A: Math. Gen. 12, 847 (1979). Solution of Einstein's equations for a line-mass of perfect fluid

[27] B. Jensen and J. Kučera, J. Math. Phys. 34, 4975 (1993). On a gravitational Aharonov-Bohm effect

[28] W. Israel, Nuovo Cimento 44B, 1 (1966); 48B, 463 (1967). Singular Hypersurfaces and Thin Shells in General Relativity.

[29] E. Poisson, A Relativist's Toolkit, (Cambridge University Press, Cambridge, U.K. 2004).

[30] P.R. Holvorcem and P.R. Letelier, Gravitation: the Spacetime Structure, SILARGVIII, Proceedings of the 8th Latin American Symposium on Relativity and Gravitation (World Scientific, Singapore, 1994) p. 315.

[31] A.B. Pavan, E. Abdalla and C. Molina, Phys. Rev. D 81, 044003 (2010). Stability, causality and quasinormal modes of cosmic strings and cylinders.

[32] B. Linet, J. Math. Phys. 27, 1817 (1986). The static, cylindrically symmetric strings in general 
relativity with cosmological constant

[33] Q. Tian, Phys. Rev. D 33, 3549 (1986). Cosmic strings with cosmological constant.

[34] J.B. Griffiths and N.O. Santos, Class. Quan. Grav. 27, 125004 (2010). A rotating cylinder in an asymptotically locally anti-de Sitter background.

[35] M. Sakellariadou, Nucl. Phys. Proc. Suppl. 192-193, 68 (2009), Cosmic Strings and Cosmic Super Strings.

[36] B. Hartmann and P. Sirimachan, arXiv:gr-qc/1007.0863. Geodesic motion in the space-time of a cosmic string.

[37] S.G. Samko, A.A. Kilbas, O.I. Marichev, Fractional Integrals and Derivatives: Theory and Applications, (Gordon and Breach S. A. 1993) 
This figure "graph_0.jpg" is available in "jpg" format from: http://arxiv.org/ps/1105.2346v1 
This figure "graph_1.jpg" is available in "jpg" format from: http://arxiv.org/ps/1105.2346v1 
This figure "graph_2.jpg" is available in "jpg" format from: http://arxiv.org/ps/1105.2346v1 
This figure "graph_4.jpg" is available in "jpg" format from: http://arxiv.org/ps/1105.2346v1 
This figure "graph_5.jpg" is available in "jpg" format from: http://arxiv.org/ps/1105.2346v1 